\title{
Histoarquitetura, Função Endócrina e Taxa de Gravidez após Auto-Implante Ovariano Ortotópico Íntegro e Fatiado em Coelha
}

\author{
Histoarchitecture, Endocrine Function and Pregnancy Rate after Orthotopic \\ Intact and Sliced Ovarian Autologous Transplantation in the Rabbit \\ Andy Petroianu, Luiz Ronaldo Alberti, Leonardo de Souza Vasconcellos
}

\begin{abstract}
RESUMO
Objetivos: verificar a possibilidade de gestação natural em coelha, após ooforectomia total bilateral e auto-implante ovariano ortotópico (integro e fatiado), sem pedículo vascular e avaliar os aspectos morfofuncionais dos ovários reimplantados.

Métodos: foram utilizadas trinta e duas coelhas da raça Nova Zelândia Branca. No grupo controle $(G C)(n=8)$ foi realizada apenas laparotomia e laparorrafia. No grupo RI $(n=8)$, reimplantou-se o ovário na forma íntegra. No grupo $R F(n=8)$, reimplantou-se o ovário na forma fatiada e, no grupo RIF ( $n=8)$, reimplantou-se, de um lado, o ovário íntegro e, do outro lado, fatiado. A partir do terceiro mês pós-operatório, cada coelha foi colocada em gaiola junto com um macho fértil, para cópula. Dosou-se o estradiol, a progesterona, o hormônio folículo-estimulante e o hormônio luteinizante de cada animal no nono mês pós-operatório. Foram estudadas as morfologias macro e microscópica dos ovários, tubas e útero, de todos os animais. $O$ número de gestações e de filhotes nascidos em cada grupo foi avaliado por meio do teste $\chi^{2} e$ as dosagens hormonais foram comparadas pelo teste t de Student, considerando $p<0,05$ como significância.

Resultados: todas as coelhas do GC engravidaram, entre o segundo e terceiro meses após início da cópula, com número de filhotes variando entre 6 e 10 animais. Nos demais grupos, as gestações ocorreram entre o quinto e o oitavo mês pós-operatório. A porcentagem de gravidez observada foi de 37,5\% no grupo RI e 50\% nos grupos RF e RIF. Não houve diferença entre os números de filhotes dos grupos com ovários reimplantados, que foi de 1 a 4 animais. Os níveis hormonais e o estudo morfofuncional dos ovários, tubas e úteros não apresentaram diferenças entre os grupos.

Conclusões: o auto-implante ovariano ortotópico em coelhas, na forma íntegra ou fatiada, sem pedículo vascular, é viável, além de preservar as funções hormonais e a fertilidade.
\end{abstract}

PALAVRAS-CHAVE: Auto-implante ovariano. Enxerto ovariano. Infertilidade.

\section{Introdução}

O conceito de transplante ovariano na medicina reprodutiva não é novo. Os primeiros relatos foram de Robert Morris e Knauer, visando ao

Departamento de Cirurgia, Faculdade de Medicina Universidade Federal de Minas Gerais.

Correspondência:

Andy Petroianu

Av. Afonso Pena 1626, apto. 1901

30130-005 - Belo Horizonte - MG

Fone/Fax: (31) 3274-7744

e-mail: petroian@medicina.ufmg.br rejuvenescimento mediante a manutenção dos níveis dos esteróides gonadais após o climatério ${ }^{1}$. A partir da década de 1940, com o advento das pílulas de reposição hormonal, houve uma diminuição das investigações sobre o transplante ovariano. Entretanto, foi apenas no final do século XX que aumentou o interesse pelo transplante ovariano, graças aos conceitos emergentes de endocrinologia e imunologia, que possibilitaram investigação mais apurada com base em transplante experimental ${ }^{1}$.

Atualmente, esse procedimento vem sendo considerado uma alternativa viável para a manu- 
tenção hormonal fisiológica em mulheres submetidas à retirada de ovários normais por processos neoplásicos ou infecciosos pélvicos ${ }^{2,3}$.

A maioria dos trabalhos experimentais sobre implantes ovarianos visa principalmente à manutenção da função endócrina ${ }^{4,5} \mathrm{e}$ à restauração da capacidade reprodutiva ${ }^{6}$. Parrott ${ }^{7}$ já havia verificado fertilidade em cobaias após reimplante ovariano. Nos últimos anos, tornou-se possivel a manutenção da gravidez em casos de ooforectomia, com métodos distintos, tais como criopreservação de ovário, transplante de folículos ou auto-implante ovariano $^{8,9}$.

Ainda são poucos os estudos nos quais a recuperação da função ovariana após transplante de folículos possibilitou índices reprodutivos adequa$\operatorname{dos}^{10}$. Segundo a maioria dos autores, o pedículo vascular em casos de auto-implante ovariano é fundamental para a viabilidade do órgão ${ }^{11}$, já que os implantes avasculares que preservam a função endócrina acompanham-se de índices gestacionais inexpressivos ${ }^{12-14}$.

Os objetivos do presente trabalho foram verificar a ocorrência de gestação natural em coelhas e avaliar aspectos morfológicos e endócrinos de implantes ovarianos autógenos, fixados em posição ortotópica (no mesmo local de onde o ovário foi retirado), na forma íntegra e fatiada, sem pedículo vascular.

\section{Material e Métodos}

Este trabalho foi realizado de acordo com as recomendações das Normas Internacionais de Proteção aos Animais ${ }^{15}$ e do Código Brasileiro de Experimentação Animal (1988), tendo sido aprovado pela Comissão de Ética do Departamento de Cirurgia da Faculdade de Medicina da Universidade Federal de Minas Gerais (UFMG).

Foram estudados 42 coelhos (Oryctogalus cuniculus) da raça Nova Zelândia Branca, sendo 32 fêmeas e dez machos sabidamente férteis. No início do experimento, todos os coelhos estavam com 4 meses de idade, sexualmente maduros e com peso variando de 2.450 a $2.700 \mathrm{~g}$. Os animais, provenientes do Biotério Central da Escola de Veterinária da UFMG foram mantidos em gaiolas individuais, onde receberam ração e água à vontade. No pré-operatório, todos permaneceram em adaptação e observação do estado de saúde por 20 dias. Após este período os animais foram mantidos em jejum pré-operatório de 12 horas.

As coelhas foram aleatoriamente distribuídas em dois grupos: grupo controle $(\mathrm{GC})(\mathrm{n}=8)$ : apenas laparotomia mediana e laparorrafia e grupo reimplante $(G R)(n=24)$ : laparotomia mediana e ooforectomia bilateral. Os animais ooforectomizados foram distribuídos em três subgrupos $(n=8)$, de acordo com o método usado para reimplante ovariano: Subgrupo GRI ( $n=8)$ : integro bilateral; subgrupo GRF $(n=8)$ : fatiado bilateral; subgrupo GRIF $(n=8)$ : integro de um lado e fatiado do outro lado.

Todos os animais receberam antibioticoprofilaxia com cefadroxil (Cefamox; Bristol-MyersSquibb, São Paulo) na dose de $50 \mathrm{mg} / \mathrm{kg}$ dissolvida em $10 \mathrm{~mL}$ de leite e administrada por cateter orogástrico 30 minutos antes do procedimento cirúrgico.

A anestesia foi induzida com injeção intramuscular na região glútea de cloridrato de ketamina (Ketalar ${ }^{\circledR}$, Pfizer, São Paulo) a 5\%, na dose de $50 \mathrm{mg} / \mathrm{kg}$. Quando necessário, foi aplicado adicionalmente um quarto da dose inicial do anestésico $^{12,20}$. Durante todo o período anestésico, foram observadas as freqüências cardíaca e respiratória, além da movimentação voluntária dos coelhos, com vista a detectar possiveis complicações.

Após tricotomia do abdome, realizou-se antisepsia com solução de iodopolivinilpirrolidona $\left(\mathrm{PVPI}^{\circledR}\right)$ degermante seguida por solução alcoólica de iodo a $2 \%$. A anti-sepsia foi seguida pela colocação de campos operatórios.

Após a laparotomia mediana infra-umbilical, identificação do útero, das tubas uterinas e dos ovários, os animais do GC $(n=8)$ foram fechados com suturas contínuas em dois planos: aponeurose, com fio de poliglactina 2-0 (Vicryl ${ }^{\circledR}$, Ethicon, São Paulo) e pele, com náilon 3-0 (Nylon ${ }^{\circledR}$, Ethicon, São Paulo). Nos animais do GR $(n=24)$, realizou-se ooforectomia bilateral, preservando-se a integridade das tubas uterinas. Em seguida, os ovários foram reimplantados nos mesmos locais de onde foram retirados, utilizando fio náilon 5-0 de acordo com os subgrupos: GRI - reimplante bilateral de ovários íntegros; GRF - reimplante bilateral de ovários fatiados longitudinalmente em três segmentos de $2 \mathrm{~mm}$ de espessura; GRIF reimplante de ovário íntegro de um lado e fatiado do outro, aleatoriamente.

Após o ato cirúrgico, as coelhas receberam ração e água previamente filtrada em recipientes próprios, ad libitum, e foram mantidas em gaiolas individuais, em condições adequadas de higiene, ventilação e iluminação.

Decorridos três meses da cirurgia, as fêmeas foram colocadas diariamente com um macho diferente sabidamente fértil para cópula, durante outros seis meses. A troca dos machos foi aleatória e ininterrupta até perceber-se a gravidez na coelha. Nessa situação a fêmea era mantida 
sem companhia em sua gaiola até o término da gestação. Registraram-se o número de gestações e o número de filhotes em cada grupo, bem como qualquer complicação gestacional que pudesse ser observada. As coelhas do GC foram expostas ao acasalamento, seguindo a mesma conduta descrita para as fêmeas com ovários reimplantados.

Seis meses após o início do acasalamento, foram colhidas amostras sangüineas, por punção da veia cava abdominal, para dosagens de estradiol, progesterona, hormônio folículo-estimulante (FSH) e hormônio luteinizante (LH), pouco antes do sacrifício do animal. Os valores hormonais obtidos nas coelhas do GC foram considerados como valores de referência para os demais grupos, tendo em vista que não houve estudo hormonal prévio nas coelhas operadas. A quantificação hormonal seguiu o método de imunofluorimetria para os hormônios gonadais e hipofisários. Nesse método, a substância a ser analisada reage com anticorpos previamente marcados na solução. Anticorpos residuais se ligam aos antígenos ligados à fase sólida. Esse complexo é lavado, e a intensidade de fluorescência obtida é inversamente relacionada à substância em análise. Esse método é adaptado para dosagens de haptenos e proteínas complexas. A principal vantagem da imunofluorimetria é a redução da interferência por substâncias naturalmente fluorescentes em amostras sangüineas, que são eliminadas durante o processo de separação das fases sólidas e líquidas.

Ao final do período de acompanhamento, após o nascimento dos filhotes ou decorridos nove meses, nas coelhas que não levaram a gestação a termo, todas as coelhas foram mortas com dose inalatória letal de éter, após anestesia profunda com ketamina (50 mg/kg). As cavidades torácica e abdominal, bem como seus órgãos, foram cuidadosamente estudados por meio de toracolaparotomia mediana. Retiraram-se o útero, os ovários e as tubas uterinas de todas as coelhas. Fragmentos de $5 \mu \mathrm{m}$ foram obtidos de cada estrutura, sendo parte deles fixados em solução de Bouin e outra parte em solução de formaldeído a 4\% (formol a $10 \%)$. Em seguida, os fragmentos fixados foram preparados para inclusão em parafina, sendo mergulhados consecutivamente em álcool a 70\%, álcool a 80\%, álcool a 90\% e álcool absoluto, durante uma hora para cada solução. O álcool absoluto foi trocado duas vezes, totalizando três horas de contato com os fragmentos, que então foram transferidos para recipientes com xilol, onde ficaram durante duas horas para diafanização, sendo essa solução trocada após a primeira hora. Em segui$\mathrm{da}$, foram alocados em recipientes com parafina e levados para uma estufa a vácuo onde permaneceram por uma hora. Na próxima etapa, os fragmentos foram incluídos em blocos de parafina histológica (Histosec ${ }^{\circledR}$ ). Posteriormente, foram feitos cortes teciduais de $4 \mu \mathrm{m}$ nos blocos de parafina, utilizando micrótomo rotativo (LEICA, modelo RM2125, Alemanha). Os cortes foram fixados sobre lâminas de vidro e corados com hematoxilina e eosina (HE) para estudo histológico de rotina. A viabilidade ovariana foi avaliada baseada nos seguintes parâmetros: presença de angiogênese, qualificação dos folículos ovarianos e presença de corpos lúteos.

Foram utilizados os testes $\chi^{2}$ com correção de Yates para pequenas amostras para comparar o número de gestações bem sucedidas e o número de filhotes, e o teste $t$ de Student para comparar as dosagens hormonais (estradiol, progesterona, FSH e LH). As diferenças foram consideradas significativas para valores correspondentes a $\mathrm{p}<0,05^{18}$. Os testes estatísticos foram realizados utilizando o software Prism $^{\circledR}$ versão 3-0 e Epi-Info ${ }^{\circledR}$ versão 6.04 .

\section{Resultados}

Todos os animais recuperaram-se espontaneamente das cirurgias e sobreviveram durante os nove meses do experimento, sem intercorrências.

A Tabela 1 mostra os valores hormonais obtidos de todos os animais. Hormônios ovarianos foram detectados em todas as coelhas com ovários reimplantados, mesmo naquelas em que não se verificou gestação. Não houve diferença entre as médias dos valores hormonais dos diversos subgrupos e as do GC ( $p>0,05)$.

Tabela 1 - Distribuição dos animais segundo os valores hormonais (média e desvio padrão da média) dosados nas coelhas controle e submetidas a reimplante ovariano ortotópico.

\begin{tabular}{lcccc}
\hline Grupos & $\begin{array}{c}\text { Estradiol } \\
(\mathrm{pg} / \mathrm{mL})\end{array}$ & $\begin{array}{c}\text { Progesterona } \\
(\mathrm{pg} / \mathrm{mL})\end{array}$ & $\begin{array}{c}\text { FSH } \\
(\mathrm{UI} / \mathrm{L})\end{array}$ & $\begin{array}{c}\text { LH } \\
(\mathrm{UI} / \mathrm{L})\end{array}$ \\
\hline GC & $2,7 \pm 0,4$ & $104,2 \pm 2,5$ & $0,2 \pm 0,1$ & $0,2 \pm 0,1$ \\
GRI & $2,5 \pm 0,9$ & $109,3 \pm 5,5$ & $0,3 \pm 0,2$ & $0,3 \pm 0,2$ \\
GRF & $2,8 \pm 0,7$ & $105,4 \pm 3,5$ & $0,2 \pm 0,1$ & $0,2 \pm 0,1$ \\
GRIF & $2,9 \pm 0,8$ & $106,1 \pm 3,3$ & $0,3 \pm 0,2$ & $0,2 \pm 0,1$ \\
\hline
\end{tabular}

GC - Grupo controle.

GRI - Reimplante ovariano íntegro.

GRF - Reimplante ovariano fatiado.

GRIF - Reimplante íntegro de um lado e fatiado do outro.

FSH - Hormônio folículo-estimulante.

LH - Hormônio luteinizante. 
Na segunda laparotomia, observou-se que a cavidade abdominal das coelhas do GC apresentavam aspecto normal. O exame macroscópico dos órgãos reprodutores mostrou ovários em topografia normal com preservação de sua parte externa e ausência de fibrose ou reações inflamatórias aparentes. O útero e as tubas das coelhas também não apresentaram alterações anatômicas. Observaram-se em duas coelhas (GC3 e GC7) aderências frouxas entre o intestino delgado e a parede abdominal.

O exame macroscópico das coelhas do GR que engravidaram mostrou ovários com preservação de sua parte externa e ausência de fibrose ou reações inflamatórias. Todavia, nas coelhas que não engravidaram encontrou-se hidrossalpinge em dois animais (GRI3 e GRF6), além de múltiplas aderências locais entre ovário, tuba e intestino nas outras duas coelhas (GRF2 e GRIF5). Não se observaram diferenças entre os ovários integros e os fatiados. O útero de todos os animais tinha aspecto normal. O restante da cavidade abdominal permaneceu sem alterações. Não houve alterações macroscópicas nos demais órgãos abdominais.

À análise histológica dos órgãos reprodutores das coelhas do GC encontrou-se mesossalpingite em uma coelha (GC2). Não foram observadas outras alterações ovarianas. Em uma outra coelha (GC4) foi identificada hipotrofia tubária à direita. O útero mostrou-se de aspecto normal, com tecido endometrial típico das várias fases de proliferação.

No GR, a histoarquitetura de todos os ovários reimplantados foi preservada. Não foram encontradas diferenças entre os ovários reimplantados integros e fatiados. A viabilidade ovariana foi confirmada pela presença de angiogênese, folículos em diferentes estádios de maturação e luteinização, bem como de cistos foliculares. Não houve sinais de isquemia ou necrose. As tubas das coelhas que engravidaram mostraram aspecto semelhante ao GC, com microvilosidades bem desenvolvidas e ausência de sinais inflamatórios ou degenerativos. Todavia, em quatro das 13 coelhas que não engravidaram foram encontradas alterações histológicas, com hidrossalpinge e múltiplas aderências locais entre ovário, tuba e intestino. A histoarquitetura uterina de ambos os grupos foi mantida, com endométrio em várias fases de proliferação, compativeis com o ciclo estral normal, indicando adequada produção hormonal.

Quanto à fertilidade, todas as coelhas do GC engravidaram, metade após dois meses e a outra metade três meses após o início dos cruzamentos. O número de filhotes variou entre 6 e 10 animais.
$\mathrm{O}$ índice de fertilidade do GR foi inferior ao encontrado no GC $(\mathrm{p}<0,05)$. No GRI, três coelhas $(37,5 \%)$ levaram sua gestação a termo. Os partos ocorreram, em duas coelhas no $5^{\circ}$ mês pós-operatório, com o nascimento de dois e três filhotes, respectivamente, e em outra, no $6^{\circ}$ mês pós-operatório, com o nascimento de um filhote (Tabela 2).

Tabela 2 - Distribuição dos animais segundo o número (n) e a porcentagem de coelhas com gestações a termo em cada grupo.

\begin{tabular}{lccc}
\hline Grupo & $\mathbf{n}$ & $\%$ & $\mathbf{p}($ em relação a GC) \\
\hline GC & 8 & 100,0 & \\
GRI & 3 & 37,5 & 0,121 \\
GRF & 4 & 50,0 & 0,280 \\
GRIF & 4 & 50,0 & 0,28 \\
\hline
\end{tabular}

GC - Grupo controle.

GRI - Reimplante ovariano íntegro.

GRF - Reimplante ovariano fatiado.

GRIF - Reimplante íntegro de um lado e fatiado do outro.

No GRF, quatro coelhas (50\%) levaram a sua gestação a termo. Os partos ocorreram, em uma coelha no $5^{\circ}$ mês pós-operatório, com o nascimento de quatro filhotes; em outra, no $6^{\circ}$ mês pós-operatório, com nascimento de um filhote, e nas outras duas, no $7^{\circ}$ mês pós-operatório, sendo uma com dois filhotes e a outra com três filhotes (Tabela 2).

No GRIF, o índice de gestação a termo também foi de $50 \%$. Os partos ocorreram, em uma coelha no $5^{\circ}$ mês pós-operatório, com o nascimento de um filhote; em outra, no $6^{\circ}$ mês pósoperatório, com nascimento de três filhotes, e nas outras duas, no $8^{\circ}$ mês pós-operatório, sendo uma com um filhote e a outra com dois filhotes (Tabela 2).

Não houve diferenças entre o GC e os demais subgrupos em relação ao número de gestações (GRI ( $p=0,121)$, GRF ( $p=0,28)$, GRIF $(p=0,28)$ ).

\section{Discussão}

Diversas técnicas experimentais de autoimplante ovariano vêm sendo pesquisadas ${ }^{19-21}$. A multiplicidade de espécies animais e de métodos estudados para a preservação da função ovariana resulta em informações conflitantes quanto à eficácia da manutenção hormonal ${ }^{20-22}$. Optou-se pelo emprego de coelha como animal de experimentação a partir da observação de sua boa capacidade reprodutiva e da simplicidade do procedimento cirúrgico sem a necessidade de material especial. Foi considerada a experiência referente a transplante de órgãos e tecidos ${ }^{23,24}$. 
No presente trabalho, o objetivo não foi apenas preservar a função hormonal do ovário e diminuição dos efeitos indesejáveis do climatério precoce $^{2,3}$, mas também verificar a capacidade reprodutiva. Na natureza e no biotério da UFMG, o número de filhotes por gestação em coelhas varia de 3 até 8 filhotes, com média de 5 . No presente estudo, não houve diferença entre o número de filhotes em relação ao GC e os submetidos a cirurgia dos ovários. Além disso, o estudo microscópico não mostrou evidências que sugerissem alterações na função hormonal, como por exemplo, atrofia ou hiperplasia endometrial, fato esse comprovado também pelas dosagens de estradiol e progesterona, que se mantiveram dentro da faixa de normalidade. Não houve diferença entre o tempo para engravidar em relação aos subgrupos estudados e nem desses com o habitual em animais em cativeiro.

O auto-implante ovariano ortotópico avascular foi um bom método para completa recuperação das funções ovarianas hormonal e reprodutiva. O suprimento sangüíneo é fator importante para manter a vitalidade tanto dos auto-implantes quanto dos transplantes de ovário.

Os resultados do presente experimento confirmam que a função ovariana pode ser preservada após castração seguida de implante ovariano sem a realização de anastomose vascular em coelhos. Esse fato também fora constatado previamente em ratos ${ }^{25-27}$. A ausência de anastomose vascular não interferiu na vitalidade dos ovários, que mantiveram neoformação vascular adequada. Não foram detectadas complicações decorrentes da falta do restabelecimento vascular cirúrgico, pois nenhum dos animais apresentou sinais de isquemia ovariana. Esses achados contradizem alguns autores que consideram fundamental a revascularização cirúrgica dos enxertos ovarianos para a manutenção de sua funcionalidade ${ }^{11,18}$.

Por outro lado, existem estudos que constataram ser a anastomose do pedículo vascular desnecessária para a manutenção da vitalidade e função ovariana. A intensa neovascularização que se forma em torno do ovário parece ser suficiente para conservar sua vitalidade ${ }^{4,5}$. Os elementos responsáveis pela estimulação da angiogênese ainda permanecem desconhecidos ${ }^{4}$. O tecido ovariano é uma rica fonte de fatores angiogênicos, que estimulam a rápida migração de células endoteliais nos casos de transplantes, o que leva ao restabelecimento da circulação sangüínea ${ }^{4,5}$. Dissen et al. ${ }^{9}$ implantaram córtex ovariano adjacente à veia jugular, e verificaram revascularização do enxerto 48 horas após o procedimento. Esse fenômeno acompanhou-se de aumento na expres- são de agentes angiogênicos específicos, principalmente do fator de crescimento derivado do endotélio. Esses autores também sugeriram que a secreção de gonadotropinas desempenha papel significativo na resposta vascular.

Dentre as gonadotropinas hipofisárias, o FSH parece ser o mais importante, devido ao estímulo à mitose celular da granulosa e à inibição de sua apoptose. O tratamento de folículos antrais de ratos com doses crescentes de FSH causam supressão da fragmentação do DNA dose-dependente, atingindo a supressão máxima de $60 \%{ }^{18}$. Em decorrência da privação de fatores mitogênicos e tróficos, como as gonadotropinas e os esteróides ovarianos, há lesão por apoptose das células do ovário. A dosagem de FSH manteve-se dentro dos limites da normalidade nos grupos estudados.

Um fator descrito como de importância para a sobrevida e desenvolvimento folicular após a retirada cirúrgica dos ovários é a temperatura média de conservação do tecido. Ao contrário de outros modelos de transplante de órgãos, o tecido ovariano mantido a temperaturas em torno de $37^{\circ} \mathrm{C}$ mostra melhor conservação se comparado ao armazenamento em gelo. É possivel que os oócitos primordiais e folículos primários tenham a mesma sensibilidade a temperaturas baixas, assim como os oócitos e folículos maduros ${ }^{8}$. Durante o ato operatório, tomou-se o cuidado de realizar o reimplante dos ovários logo após a ooforectomia, com o intuito de se manter a temperatura ovariana o mais próximo da fisiológica, minimizando as possiveis perdas de células ovarianas.

A avaliação macroscópica e microscópica não mostrou diferenças quanto à preservação ovariana quando se implantava na forma íntegra ou fatiada. Por outro lado, em estudo prévio com ratas, os resultados hormonais foram melhores no grupo de reimplante ovariano fatiado ${ }^{25-27}$. Contudo, essa diferença não foi verificada na coelha. Cabe, portanto, considerar que o implante ovariano fatiado possa ser mais eficaz do que o íntegro. Diante da falta da uniformidade entre os resultados das duas espécies estudadas por nós e aparente ausência de subsídios da literatura para essa questão, é fundamental prosseguir nesta linha de pesquisa, em busca da resposta a esse problema.

Embora as gestações das coelhas do GRI (integro bilateral) tenham sido em menor número do que a dos GRF (fatiado bilateral) e GRIF (integro de um lado e fatiado do outro), essa diferença não foi significativa. Cabe ressaltar que a freqüência gestacional obtida neste trabalho e em outros estudos desta linha de pesquisa ${ }^{25,26}$ foi superior à alcançada por outros autores ${ }^{12,17}$.

A infertilidade de algumas coelhas pode ser 
atribuída à presença de aderências tubo-ovarianas e outros fenômenos inflamatórios presente nas tubas uterinas. Embora não tenha sido evidenciada gravidez, a função endócrina ovariana dessas coelhas também se manteve preservada. É pertinente supor a ocorrência de gestação não identificada ao longo do período de acompanhamento que foi interrompida com abortamento. Os abortamentos ocorrem naturalmente em animais e a literatura mostra uma grande variedade de etiologias para esses abortamentos, incluindo alterações genéticas, anatômicas, hormonais e infecciosas, além de influência ambiental.

O período da coleta sangüínea para as dosagens hormonais coincidiu com a fase de puerpério em todas as coelhas do GC. O mesmo não ocorreu com os animais dos grupos submetidos aos procedimentos, o que explica a maior variação hormonal entre os animais de mesmo subgrupo. A dosagem de FSH não mostrou diferença significante entre os grupos controle e ooforectomizado. Esse fato mostra que os ovários reimplantados mantinham produção hormonal de estradiol dentro da normalidade, já que por mecanismo de retroalimentação, qualquer diminuição da produção de estradiol estimularia a liberação do FSH.

A secreção basal de LH é suprimida pela combinação da progesterona secretada pelo corpo lúteo e do estradiol derivado dos folículos antrais ${ }^{14}$. Como a concentração de LH manteve-se dentro dos limites da normalidade nos grupos de reimplante e de transplante, tal fato comprova adequada produção hormonal nos ovários estudados.

Concluindo, o auto-implante ovariano ortotópico, na forma integra ou fatiada, sem preservação do pedículo vascular, é viável e mantém as funções de produção hormonal e a fertilidade.

\section{ABSTRACT}

Purpose: to assess the natural pregnancy rates in rabbits submitted to bilateral oophorectomy and orthotopic autologous (both intact and sliced) ovarian transplantation without vascular pedicle and to verify the morphofunctional aspects of reimplanted ovaries.

Method: thirty-two female New Zealand White rabbits were studied. The ovaries were removed and orthotopically replaced without vascular anastomosis. In the control group $(G C)(n=8)$, only laparotomy was carried out. In the RI group $(n=8)$ intact ovaries were reimplanted in both sides. In the $R F$ group $(n=8)$ the ovaries were sliced and orthotopically reimplanted. In the RIF group $(n=8)$, in one side the intact ovary and in the other side the sliced ovary was reimplanted.
Three months later, the animals were paired with sexually mature males for copulation. Estradiol, progesterone, follicle stimulating hormone and luteinizing hormone levels were assessed nine months after surgery. Histologic study of the transplanted ovaries, tubes and uteri was carried out, and the number of pregnancies and animals per litter in each group was taken into account as well. The $\chi^{2}$ test compared the pregnancy and the litters between the groups. Student's $\mathrm{t}$ test compared the hormone determinations. Significance was set at $p<0.05$.

Results: pregnancies occurred in all rabbits of the $G C$, between the second and third months after copulation, with 6 to 10 animals per litter for animal. In the other groups, the pregnancies occurred between the fifth and eighth postoperative month. Pregnancies occurred in $37.5 \%$ of rabbits in group RI, and in 50\% of the RF and RIF groups. There was no difference between the number of animals per litter in the reimplanted groups, with 1 to 4 animals per litter for each animal. Hormone levels and histology confirmed the vitality of all ovaries.

Conclusions: intact or sliced orthotopic autologous ovarian transplantation without vascular pedicle is viable in rabbits, and preserves the hormonal and fertile functions.

KEYWORDS: Ovary. Ovarian transplantation. Ovarian graft. Inferlility.

\section{Agradecimentos}

Agradecemos ao CNPq e à FAPEMIG pelos auxílios financeiros que permitiram a realização deste trabalho.

\section{Referências}

1. Trounson A, Bongso A. Fertilization and development in humans. Curr Top Dev Biol 1996; 32:59-101.

2. Chapman RM, Sutcliffe SB, Malpas JS. Cytotoxic induced ovarian failure in women with Hodgkin's disease. I. Hormone function. JAMA 1979; 242:187781 .

3. Resende CAL, Gobbi H, Geber S. Aspectos embriológicos, histológicos, anatômicos e fisiológicos dos ovários. In: Coronho V, Petroianu A, Santana EM, Pimenta LG, editores. Tratado de Endocrinologia e Cirurgia Endócrina. 1 ${ }^{\text {a }}$ ed. Rio de Janeiro: Guanabara Koogan; 2001. p.1060-95.

4. Weissman A, Gotlieb L, Colgan T, Jurisicova A, Greenblatt EM, Casper RF. Preliminary experience with subcutaneous human ovarian cortex transplantation in the NOD-SCID mouse. Biol Reprod 1999; 60:1462-7. 
5. Nisolle M, Casanas-Roux F, Qu J, Motta P, Donnez J. Histologic and ultrastructural evaluation of fresh and frozen-thawed human ovarian xenografts in nude mice. Fertil Steril 2000; 74:122-9.

6. Dziuk PJ, Parvizi N, Ellendorff F. Concentrations of free steroids in the jugular and hepatic portal veins of pigs after ingestion of testosterone, estrogen, or progesterone or transplantation of ovaries to the intestine. Domest Anim Endocrinol 1999; 17:29-38.

7. Parrott DM. Orthotopic ovarian grafts in the golden hamster. J Endocrinol 1959; 19:126-38.

8. Aubard Y, Newton H, Scheffer G, Gosden R. Conservation of the follicular population in irradiated rats by the cryopreservation and orthotopic autografting ovarian tissue. Eur J Obstet Gynecol Reprod Biol 1998; 79:83-7.

9. Dissen GA, Lara HE, Fahrenbach WH, Costa ME, Ojeda SR. Immature rat ovaries become revascularized rapidly after autotransplantation and show a gonadotropin-dependent increase in angiogenic factor gene expression. Endocrinology 1994; 134: 1146-54.

10.Gosden RG. Restitution of fertility in sterilized mice by transferring primordial ovarian follicles. Hum Reprod 1990; 5:499-504.

11. Namba Y, Yamamoto M, Atrishima K, Eguchi Y. Suppressive effect of perinatal tests on the differentiation of fetal ovaries transplanted into adult males in the rat. J Anat 1997; 191:31-8.

12. Green CJ, Simpkin S, Grimaldi G, Johnson A. Pregnancy after autografting and allografting vascularized ovaries and en bloc vascularized ovaries with adnexa in rabbits. Br J Obstet Gynaecol 1982; 89:645-51

13.Von Eye Corleta H, Corleta O, Capp E, Edelweiss MI. Subcutaneous autologous ovarian transplantation in Wistar rats maintains hormone secretion. Fertil Steril 1998; 70:16-9.

14. Candy CJ, Wood MJ, Whittingham DG. Follicular development in cryopreserved marmoset ovarian tissue after transplantation. Hum Reprod 1995; 10:2334-8.

15.Hoff C. Sounding board. Immoral and moral uses of animals. N Engl J Med 1980; 302:115-8.

16. Altman DG, Gardner MJ. Means and their differences. In: Altman DG, Machin D, Bryant TN, Gardner MJ, editors. Statistics with Confidence. $1^{\text {st }}$ ed. London: Br Med J Books; 2000. p.28-35.
17.Nugent D, Meirow D, Brook PF, Aubard Y, Gosden RG. Transplantation in reproductive medicine: previous experience, present knowledge and future prospects. Hum Reprod Update 1997; 3:267-80.

18.Callejo J, Jauregui MT, Valls C, Fernandez ME, Cabre S, Lailla JM. Heterotopic ovarian transplantation without vascular pedicle in syngeneic Lewis rats: six-month control of estradiol and follicle-stimulating hormone concentrations after intraperitoneal and subcutaneous implants. Fertil Steril 1999; 72:513-7.

19.Parkes AS. The rise of reproductive endocrinology, 1926-1940. J Endocrinol 1966; 34:xx-xxxii.

20. Rone JD, Halvorson LM, Goodman AL. Ovarian angiogenesis in rabbits: endotheliotrophic chemoattractant activity from isolated follicles and dispersed granulosa cells. J Reprod Fertil 1993; 97:359-65.

21.Parks JE, Ruffing NA. Factors affecting low temperature survival of mammalian oocytes. Theriogenology 1992; 37:59-72.

22.Sanders JE, Buckner CD, Amos D, et al. Ovarian function following marrow transplantation for aplastic anemia or leukemia. J Clin Oncol 1988; 6:813-8.

23. Vasconcellos LS, Petroianu A, Alberti LR, Leite JM, Castro LPF. Gestação após auto-implante ovariano avascular em coelha. Reprod Clim 2002; 16:273-6.

24.Petroianu A, de Souza Vasconcellos L, Alberti LR, Fonseca de Castro LP, Barbosa Leite JM. Natural pregnancy in rabbits that underwent oophorectomy and orthotopic allogeneic or autologous ovarian transplantation. Fertil Steril 2002; 77:1298-9.

25.Alberti LR, Vasconcellos LS, Barbosa JF, Petroianu A. Implante autólogo ovariano no omento maior: estudo experimental. Rev Bras Ginecol Obstet 2002; 24:187-92.

26.Alberti LR, Vasconcellos LS, Petroianu A, Nunes MB. Avaliação morfofuncional do auto-implante ovariano no retroperitônio. Medicina (Ribeirão Preto) 2002; 35:30-5.

27.Vasconcellos LS, Alberti LR, Petroianu A, Nunes MB. Viabilidade e função endócrina de autoimplantes ovarianos com drenagem pela veia porta ou cava. Reprod Clim 2001; 16:259-63. 